\title{
THE PROPOSED ACCELERATOR FACILITY FOR LIGHT ION CANCER THERAPY IN HEIDELBERG
}

\author{
H. Eickhoff, D. Böhne, J. Debus, Th. Haberer, G. Kraft, M. Pavlovic \\ GSI Darmstadt
}

\section{INTRODUCTION}

During the last four years GSI has developed a procedure for cancer treatment by means of the intensity controlled rasterscan-method. This method includes active variations of beam parameters during the treatment session and the integration of 'on-line' PET monitoring.

Starting in 1997 several patients have been successfully treated within this GSI experimental cancer treatment program. The developments and experiences of this program accompanied by intensive discussions with the medical community led to a proposal for a hospital based light ion accelerator facility for the clinic in Heidelberg. [1]

The main characteristics of this facility are the application of the rasterscan method with active intensity, energy-, and beamsize- variation in combination with the usage of isocentric light ion gantries. The accelerator is designed to accelerate both low LET ions ( $\mathrm{p}, \mathrm{He}$ ) and high LET ions $(\mathrm{C}, \mathrm{O})$ to cover the specific medical requirements.

Major aspects of the design are influenced from the experiences of the GSI cancer treatment program; the requirements of this facility, however, exceed in many fields those of this GSI therapy program.

\section{REQUIREMENTS}

The basis of the accelerator concept has to satisfy the demands of the medical community for the treatment procedures. One of the key aspects of the proposed facility is the use of the intensity controlled rasterscan technique (Fig. 1), which is a novel treatment concept, developed at GSI and successfully applied within patient treatments of the GSI pilot project.

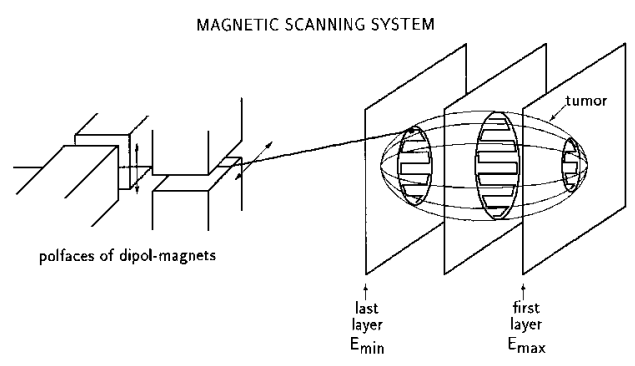

Fig. 1 Rasterscan-Method

The accelerated and slowly extracted beam enters 2 fast scanner magnets, that deflect the beam both in horizontal and vertical direction to cover the lateral dimensions of the tumor. Ionization chambers in front of the patient measure the number of ions at a specific irradiation point and control the scanner excitation. Fast multiwire proportional counters detect the position and beam width at each scanning point. When a required dose limit has been reached the beam extraction is interrupted very fast $(<0.5 \mathrm{~ms})$.

Whereas at presently existing therapy-dedicated protonand light-ion accelerators for cancer treatment the beamparameters are constant over the treatment interval, this method demands fast, active energy-variation to achieve different penetration depths and intensity-variation to minimize the treatment time [1].

The main requirements of the proposed facility were intensively discussed with radiotherapists and biophysicists and can be summarized as follows:

Table. 1: Therapy requirements

- intensity-controlled rasterscan method

- treatment both with low and high LET-ions

- relatively fast change of ion species

- wide range of particle intensities

- integration of isocentric gantries

- 3 treatment areas to treat a large number of patients

- ion-species : $\mathrm{p}, \mathrm{He}, \mathrm{C}, \mathrm{O}$

- ion-range (in water) : $20-300 \mathrm{~mm}$

- ion-energy : $50-430 \mathrm{MeV} / \mathrm{u}$

- extraction-time : $1-10 \mathrm{~s}$

- beam-diameter :4-10 mm (hor., vert.)

- intens. (ions/spill) : $1 * 10^{6}$ to $4 * 10^{10}$ (dependent. upon ion species)

\section{LAYOUT OF THE ACCELERATOR FACILITY}

Fig. 1 shows the cross section of ground floor of the building (about $70 * 60 \mathrm{~m}^{2}$ ), which gives an impression of the accelerator-sections, the position of the patients preparation areas, local control rooms and various laboratories. Additional space for housing the power supplies and further technical infrastructure is available at another floor of this building.

The following description mainly concentrates on a technical discussion of the various accelerator sections and their individual features. 


\section{a) Injector and low energy beamline}

One significant medical requirement is the demand to change the ion species used for treatment (e.g. protons ond carbon ions) within less than 30 minutes; due to this specification the installation of two ECR sources is proposed, which permanently have to be in an operational state. The ECR source is chosen, as this type provides a very stable intensity over a long time without adjustment of the source parameters. The required particle currents between $80 \mu \mathrm{A}$ (for $16 \mathrm{O}^{6+}$ and $1.2 \mathrm{~mA}$ (for $\mathrm{p}$ ) are rather conservative; beam tests of this commercially available source indicate, that both the current and the requested beam emittance can easily be achieved.

Each source branch is equipped with a 90 degree analysing magnet for the definition of the requested ion charge state and the detection of contaminations, and a solenoid and quadrupole singlet for beam matching. The extraction voltage of the ECR-source is defined to 8 $\mathrm{keV} / \mathrm{u}$.

Both branches are combined by means of a switching magnet, which leads the beam to the low energy beam transport system (LEBT) up to the linac RFQ. The LEBT contains beside a matching quadrupole triplet, a macropulse chopper and a matching solenoid an rfchopper-system to perform the requested intensity reduction up to $0.1 \%$ of the maximal ion intensity.

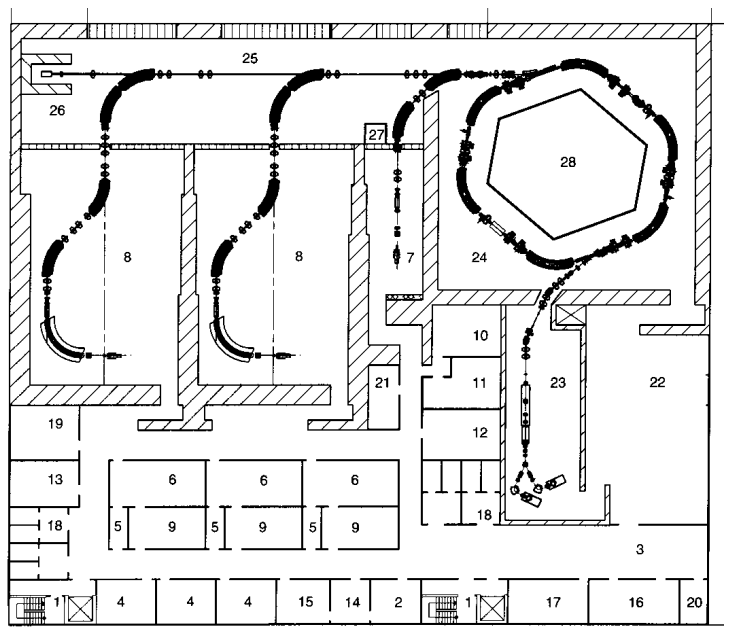

Fig. 2 Layout of the accelerator sections

\section{b) Linac, Medium energy beam transport}

A combination of RFQ and IH-linac structure with a total length of about $6 \mathrm{~m}$ is proposed to accelerate the ions up to $7 \mathrm{MeV} / \mathrm{u}$. The RF-frequency of these structures is $216 \mathrm{MHz}$. The designed pulse length is $200 \mu \mathrm{s}$, the repetition frequency $5 \mathrm{~Hz}$. The normalized beam emittance is about $0.4 \pi \mathrm{mm}$ mrad, the momentum spread $0.1 \%$.

The medium energy beam transport consists of a stripping and a matching section to the synchrotron. In addition, for multiturn injection a chopper system is provided to match the pulse from the linac.

\section{c) Synchrotron}

For the synchrotron with a circumference of about 64 meters 6 bending magnets with a maximum flux density of 1.6 T and a doublet focusing structure are proposed. 6 long drift spaces are available for the installation of injection and extraction elements and the RF-cavity. After a 15 turn injection, corresponding to an injection time of $30 \mu \mathrm{s}$, the acceleration takes place within $0.5 \mathrm{~s}$. For slow extraction with variable extraction time between 1 and $10 \mathrm{~s}$ and multiple beam extraction at the same flat top the 'transverse knock out' method is proposed.

\section{d) High energy beam transport}

The high energy beam transport system leads the slowly extracted beam either to a beam dump or distributes it to three treatment places. Just after the synchrotron extraction section a fast deflecting magnet will prohibit the beam delivery in case of interlocks.

\section{e) Treatment areas}

In order to meet the demand for a patient flow of 1000 patients/year three treatment areas are foreseen. For the first area the beam will be delivered from a horizontal beam line, similar to that used at the GSI pilot project. The beam for the second and third treatment places will be delivered by a rotating beam transport system ('isocentric gantry'). All beam lines are equipped with horizontal and vertical scanning magnets and beam dignostic devices for the intensity controlled rasterscan.

The integration of PET monitoring systems both in the horizontal and the gantry beam lines is proposed as well.

\section{f) Gantry beam lines}

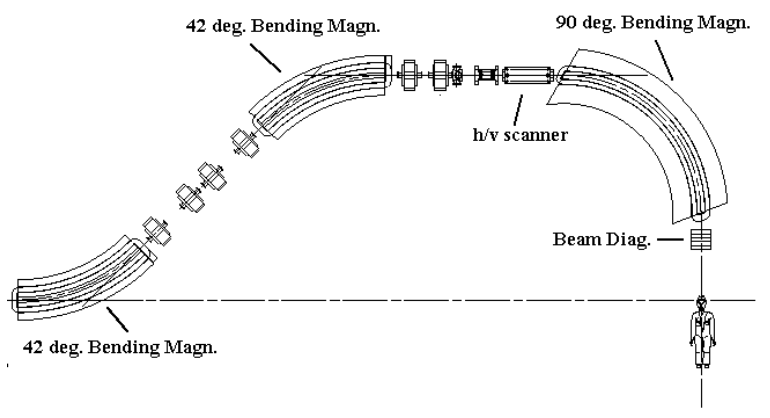

Fig. 3: Layout of the isocentric Gantry

A strong demand of the medical community is the installation of two gantry beamlines for irradiation angles between 0 and 360 degree. An isocentric gantry type with two 42 and one 90 degree bending magnets is proposed, in which the horizontal and vertical scanning magnets are located upstream before the last 90 degree deflection magnet in order to minimize the gantry-radius. The irraddiation field is $20 * 20 \mathrm{~cm}^{2}$.

The total length of this gantry is about $20 \mathrm{~m}$ and the outer radius $6.3 \mathrm{~m}$. In order to limit the concrete shielding 
the counterweight of the mechanical rotator also serves as a radiation shielding.

A special ion optics is used which provides an achromatic beam transport to the treatment location independent from the gantry angle. In addition a constant beam diameter is achieved for different horizontal and vertical beam emittances.

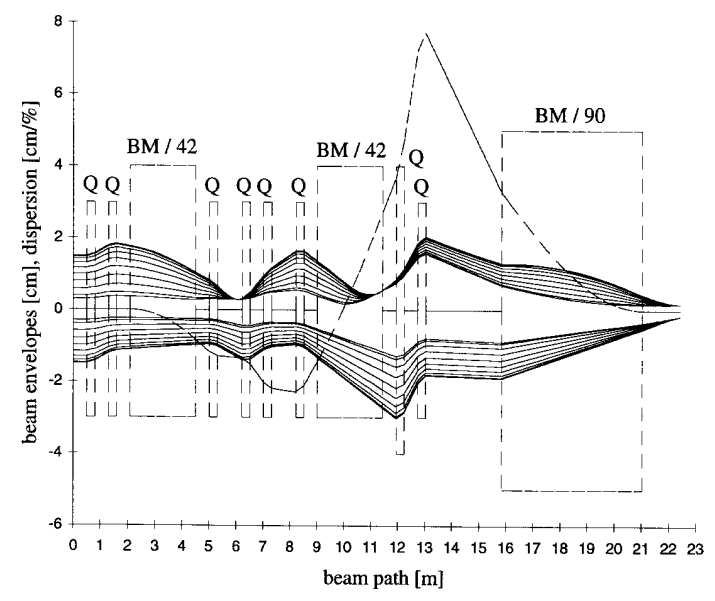

Fig. 4: Beam envelopes for gantry angles between 0 and 90 degree $\left(\varepsilon_{\mathrm{h}} / \varepsilon_{\mathrm{h}}=0.5 / 5.0 \pi \mathrm{mm} \mathrm{mrad}\right)$

(upper trace $=$ hor., lower trace $=$ vert. env. ---- = dispersion function)

\section{STATUS}

To a certain extend the proposed projects benefits from the experiences that have been achieved within the GSI pilot therapy project.

-The principles of the accelerator control based on the usage of approved settings, stored in nonvolatile memories will probably transferred. The demonstrated long term stability of the beam position (see Fig. 5) could be further improved with a moderate position feed back beam by means of the fast position monitors and the rasterscan-magnets.

-The intensity controlled rastercan-technique has been approved within a large number of treatment fractions applied to patients. Despite the strong intensity modulation of the spill the requested dose uniformity could be reliably achieved.

-The 'on-line' Positron-Emission Tomography (PET) has proved to be a valuable diagnostic tool for dose verification. The application of this method to the isocentric gantry is under investigation.

-Most of the anticipated test procedures both at the accelerator and the treatment place have been evaluated.

At GSI for the proposed facility various ion optical design studies and first design studies of critical elements have been performed. These investigations concern all accelerator sections from the source to the treatment caves. Detailed calculations of the field homogenity for the gantry 90 degree bending magnet have beeen done. Design criteria for this magnet were evaluated. In advance, dedicated studies of critical items (e.g. the gantry design) will be performed in collaboration between the GSI, the DKFZ, the Heidelberg Clinic and industries. Additional funding for the 'multifield irradiation technique' has been applied. [3] Within this application a test of the last gantry section with beam at GSI is proposed. First steps concerning the organization structures and the financing of this project have been undertaken.
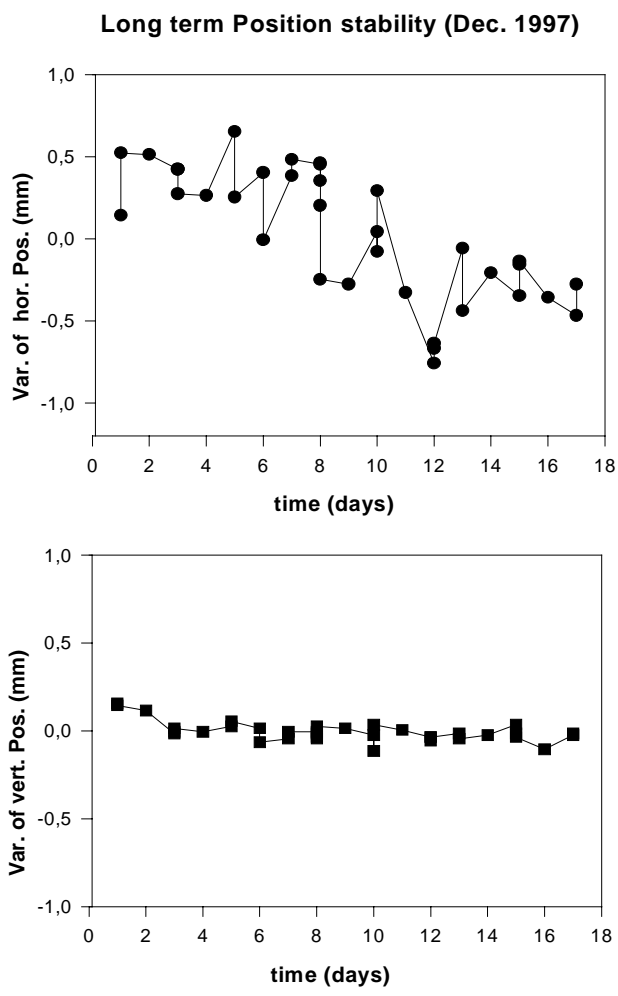

Fig. 5 Long-term position variations

It is planned to build the facility under the project leadership of the radiological clinic in Heidelberg with substantial support from industries. The know-how and the experiences of GSI shall be transferred to the industrial partners. The total project cost are assumed to be about 120 Mio DM. First patient treatments are scheduled around 2004/5 after an extensive commissioning phase of about 1 year.

[1] J. Debus et al., 'Proposal for a dedicated ion beam facility for cancer therapy', 1998

[2] H. Eickhoff et al, 'Accelerator Control For the GSI Cancer Therapy Project, EPAC 1998, 2348

[3] J. Debus et al., HGF Strategy Fund Proposal Development of intensity controlled multifieldirradiation techniques for cancer therapy with ion beams', 1999 